\title{
On the spherical collapse model in dark energy cosmologies
}

\author{
D. F. Mota and C. van de Bruck
}

\author{
Astrophysics, University of Oxford, Keble Road, OX1 3RH, UK \\ e-mail: mota@astro.ox.ac.uk; cvdb@astro.ox.ac.uk
}

Received 23 January 2004 / Accepted 30 March 2004

\begin{abstract}
We study the spherical collapse model in dark energy cosmologies, in which dark energy is modelled as a minimally coupled scalar field. We first follow the standard assumption that dark energy does not cluster on the scales of interest. Investigating four different popular potentials in detail, we show that the predictions of the spherical collapse model depend on the potential used. We also investigate the dependence on the initial conditions. Secondly, we investigate in how far perturbations in the quintessence field affect the predictions of the spherical collapse model. In doing so, we assume that the field collapses along with the dark matter. Although the field is still subdominant at the time of virialisation, the predictions are different from the case of a homogeneous dark energy component. This will in particular be true if the field is non-minimally coupled. We conclude that a better understanding of the evolution of dark energy in the highly non-linear regime is needed in order to make predictions using the spherical collapse model in models with dark energy.
\end{abstract}

Key words. cosmology: theory - cosmology: large-scale structure of Universe - cosmology: miscellaneous

\section{Introduction}

The most surprising result of observational cosmology is the discovery that high redshift supernovae are less bright than expected (Perlmutter et al. 1999; Tonry et al. 2003). The interpretation of this finding is that the expansion of the universe accelerates, instead of slowing down. The matter responsible for this accelerated expansion is dubbed "dark energy" and, according to General Relativity, needs to have negative pressure.

One important goal of cosmology is to obtain information about the nature of dark energy (see e.g., Ratra \& Peebles 2002, for a review). The physical origin of dark energy is unknown and its likely that new physics beyond the standard model of particle physics will be needed to explain its properties. Scalar fields are plausible candidates for dark energy (see e.g., Wetterich 1988a,b; Ratra \& Peebles 1988; Wetterich 1995) (these scalar fields are called quintessence fields). A distinctive feature of these models is that the properties of dark energy, such as its equation of state, generally vary during the cosmic history.

Cosmological observations probe the properties of dark energy. The anisotropy spectrum of the Cosmic Microwave Background (CMB), the shape of matter power spectrum and the distance-redshift relation are possible sources of information and will help us to distinguish between the different models of dark energy.

The behaviour of small perturbations in a scalar field and its effect on $\mathrm{CMB}$ anisotropies and structure formation has

Send offprint requests to: $\mathrm{C}$. van de Bruck,

e-mail: cvdb@astro.ox.ac.uk been investigated by a number of authors. However, the behaviour of quintessence during the gravitational collapse into the highly non-linear regime is not well understood and currently under investigation (see e.g., Bartelmann et al. 2002; Wetterich 2002; Dolag et al. 2003; Maccio et al. 2003a,b; Mainini et al. 2003a,b; Matarrese et al. 2003; Perrotta et al. 2004; Amendola 2003; Linder \& Jenkins 2003; Alcubierre et al. 2002; Guzman \& Urena-Lopez 2003, for recent work).

Usually it is assumed that there are no density fluctuations in the quintessence field on cluster scales and below. The reason for this assumption is that, according to linear perturbation theory, the mass of the field is very small (the associated wavelength of the particle is of the order of the Hubble radius) and, hence, it does not feel overdensities of the size of tenth of Mpc or smaller (Wang \& Steinhardt 1998). Let us assume for instance the particle physics candidate for dark energy, a scalar field $\phi$. In the linear regime of the small cosmological perturbations, it can be shown that, during the matter dominated epoch, perturbations in the scalar field are described by Hwang \& Noh (2001)

$\delta \ddot{\phi}+3 H \delta \dot{\phi}+\left(k^{2} / a^{2}+V^{\prime \prime}\right) \delta \phi=\dot{\phi} \delta_{\mathrm{cdm}}$

where the metric was perturbed about a flat FriedmannRobertson-Walker (FRW) metric. From Eq. (1) the effective Jeans length for linear perturbation of the scalar field is roughly given by

$\lambda_{\mathrm{J}} \approx 2 \pi / \sqrt{V_{, \phi \phi}}$

which turns out to be of the order of the horizon size (Ma et al. 1999). Thus, on scales much smaller than $\lambda_{\mathrm{J}}$ the fluctuations on the field are unimportant. This results lead people 
to assume that dark energy is an homogeneous field throughout the universe, which is not affected by structure formation on small scales. However, the source term $\dot{\phi} \delta_{\text {cdm }}$ implies that dark energy is not smoothly spread (Caldwell et al. 1998). And in spite of the perturbations in dark energy are present and grow only on scales about the horizon size and larger, the effects on the evolution of matter overdensities are indeed important and significant (Ferreira \& Joyce 1997). For instance, the spatially inhomogeneous distinctive property of quintessence, with respect to the cosmological constant $\Lambda$, has an important effect on the large-angular-scale of the CMBR (Steinhardt 2003). To study the effect of dark energy on small scales, $N$-body simulations were used and the assumptions made that quintessence perturbations are negligible. In Maccio et al. (2003b), a possible coupling of dark energy and dark matter was explicitly taken into account for the perturbations.

The assumption of neglecting the effects of matter perturbations on the evolution of dark energy (and its backreaction) at small scales is indeed a good approximation when perturbations in the metric are very small. Notice however, if the field couples explicitly to matter, such as in the coupled quintessence scenario (see e.g., Wetterich 1995; Amendola 2000), the conclusion that the Jeans length is of order the horizon size should no longer hold.

Nevertheless, even if the field is not coupled, one should be careful when extrapolating the small-scale linear-regime results to the highly non-linear regime. Then, locally the flat FRW metric is not a good approximation anymore to describe the geometry of overdense regions. It is natural to think that once a dark matter overdensity decouples from the background expansion and collapses, the field inside the cluster feels the gravitational potential inside the overdensity and its evolution will be different from the background evolution. The backreaction effects in the highly non-linear regime could influence the evolution of perturbations in dark energy considerably, which in turn influence the evolution of the matter perturbation. In Bean \& Magueijo (2002) it was suggested that the quintessence field could have an important impact in the highly non-linear regime. Due to these considerations, one may even ask if the quintessence field can be important even on galactic scales? It was speculated by Wetterich $(2001,2002)$ and by Arbey et al. (2001) that highly non-linear perturbations might indeed be important even on galactic scales. It was found that, at least in principle, the quintessence (or a scalar field) field could be responsible for the observed flat rotation curves in galaxies. In Padmanabhan \& Choudhury (2002), Padmanabhan (2002), Bagla et al. 2003 and Causse (2003) more exotic models, based on tachyon fields, have been discussed and it was argued that the equation of state is scaledependent.

If it turns out that backreaction effects of metric and density perturbations in dark matter could influence perturbations of quintessence on small scales, this could significantly change our understanding of structure formation on galactic and cluster scales in models with quintessence.

One popular model to study the non-linear growth in cold dark matter is the spherical collapse model (see e.g., Padmanabhan 1995; or Peacock 1999). The model was used first in the standard cold dark matter scenario, but later also in the cold dark matter model with cosmological constant ( $\Lambda$ CDM) by Lahav et al. (1991). Recently, the model was also used in quintessential scenarios and dark matter under the assumption that dark energy does not cluster on scales much smaller than the horizon (see e.g., Wang \& Steinhardt 1998). It was then subsequently used in order to make predictions for cosmological observations (see e.g., Weller et al. 2001; Weinberg \& Kamionkowski 2003; Battye \& Weller 2003).

The aim of this paper is two-fold. Firstly, we model dark energy as a non-minimally coupled scalar field and consider four different potentials in order to investigate how the predictions of the spherical collapse model depend on the potential. In addition, the effect of the initial conditions are investigated as well. Our second aim is to get a feel about the effects of perturbations in the quintessence field on the predictions of the spherical collapse model. In doing so, we investigate two extreme cases: in the first case we follow the literature and assume that there are no fluctuations in the quintessence field on the scales of interest. We then assume the other extreme case and assume that the field inside the overdensity collapses together with the dark matter ${ }^{1}$. As we will see, the predictions of the spherical collapse model will be quite different in this case, although the field is only slightly non-linear (with density contrasts of order one) at the time of virialisation, whereas dark matter is in the highly non-linear regime. Our results imply that the predictions of the spherical collapse model depend on the assumptions made for the clustering properties of the quintessence in that model. Thus, a better understanding of the behaviour of the scalar field in the highly non-linear regime is needed, if the spherical collapse model is used for predictions involving large scale structures, such as cluster abundances, weak and strong lensing, etc.

The paper is organised as follows: in Sect. 2 we describe briefly the spherical collapse model and write down the equations used. We also give an overview of the potentials used. In Sect. 3 we describe our assumptions and give the results of our numerical calculations. A discussion of the results and our conclusions can be found in Sect. 4.

\section{The spherical collapse model}

We consider a flat, homogeneous and isotropic background universe with scale factor $a(t)$. Since we are interested in the matter dominated epoch, when structure formation starts, we assume that the universe is filled with cold dark matter of density $\rho_{m} \propto a^{-3}$ and a dark energy fluid, with energy-density $\rho_{\phi}$. The equations that describe our background universe are (we set $\hbar=c \equiv 1$ throughout the paper):

$$
\begin{aligned}
& 3 H^{2}=8 \pi G\left(\rho_{m}+\rho_{\phi}\right) \\
& \dot{\rho}_{\phi}=-3 H\left(1+w_{\phi}\right) \rho_{\phi}
\end{aligned}
$$

where $H \equiv \dot{a} / a$ is the Hubble rate. When $w_{\phi}=-1$ then dark energy is the vacuum energy density $\Lambda$. If dark energy is a scalar field $\phi$ (quintessence), $\rho_{\phi}=\frac{1}{2} \dot{\phi}^{2}+V(\phi)$ and $P_{\phi}=\frac{1}{2} \dot{\phi}^{2}-V(\phi)$,

1 This approach was first used, in the context of varying-fine structure theories (Mota \& Barrow 2004a,b). 
where $V(\phi)$ is the scalar field potential. In this case, it is useful to re-write Eq. (4) as

$\ddot{\phi}+3 H \dot{\phi}+V^{\prime}=0$

where the prime represents a derivative with respect to $\phi$. In this paper we consider four examples of quintessential potentials:

- the double exponential potential (Barreiro et al. 2000);

$V(\phi)=M(\exp (\beta \phi)+\exp (\gamma \phi))$

- the exponential potential with inverse power (Steinhardt et al. 1999):

$$
V(\phi)=M(\exp (\gamma / \phi)-1) ;
$$

- the Albrecht-Skordis model (Albrecht \& Skordis 2000):

$$
V(\phi)=M\left(A+(\phi-B)^{2}\right) \exp (-\gamma \phi) ;
$$

- the supergravity-motivated potential (Brax \& Martin 1999):

$$
V(\phi)=M \exp \left(\phi^{2}\right) / \phi^{\gamma} \text {. }
$$

We choose the parameters in the potentials and the initial conditions in the background such that today we obtain $\Omega_{m}=$ $0.3, \Omega_{\phi}=0.7, H_{0}=100 h \mathrm{~km} /(\mathrm{Mpc} \cdot \mathrm{s})$ with $h=0.7$ and $-1 \leq w_{\phi} \leq-0.8$.

In order to study the non-linear evolution of the gravitational collapse we make use of the spherical collapse (or infall) model. Here, one considers a spherical overdense region of radius $R$ and models the interior spacetime as a FRW universe. This approach is equivalent to study the effect of perturbations to the Friedmann metric by considering spherically symmetric regions of different spatial curvature in accord with Birkhoff's theorem. Clearly, this model ignores any anisotropic effects of gravitational instability or collapse. However, the model is quite useful to learn about non-linear gravitational collapse and it is widely used as a starting point for (semi-) analytical models of large-scale structure formation.

Consider a spherical perturbation, in the cold dark matter fluid, with (spatially) constant internal density which, at an initial time, has an overdense amplitude $\delta_{\mathrm{i}}>0$ and $\left|\delta_{\mathrm{i}}\right| \ll 1$. The cold dark matter density inside the cluster is then initially $\rho_{\text {cdm }}=\rho_{m}\left(1+\delta\left(R_{\mathrm{i}}, t_{\mathrm{i}}\right)\right)$, where $R_{\mathrm{i}}$ is the initial radius of the overdensity. The dark energy fluid density inside the overdensity, $\rho_{\phi_{\mathrm{c}}}$, will initially be considered the same as the background one $^{2}$. At early times the sphere expands along with the background, but with a slightly different expansion rate. For a sufficiently large $\delta_{\mathrm{i}}$, gravity prevents the sphere from expanding for ever: at one point, the overdensity will stop expanding and start to collapse. Three characteristic phases during the evolution can then be identified:

- turnaround: the sphere breaks away from the general expansion and reaches a maximum radius;

- collapse: if only gravity is significant, the sphere will then collapse towards a central singularity where the densities of the matter fields would formally go to infinity. In practise, pressure and dissipative physics intervene well before this singularity is reached and convert the kinetic energy of collapse into random motions;

\footnotetext{
${ }^{2}$ This assumption will be made throughout the paper.
}

- virialisation: dynamical equilibrium is reached and the system becomes stationary: the radius of the system the energy of the different components are constant.

In the spherical collapse model, due to its symmetry, the only independent coordinates are the radius of the overdensity and time. Also, as is standard practise when using this model, we consider that there are no shell-crossing which implies mass (of cold dark matter) conservation inside the overdensity and independence of the radius coordinate (Padmanabhan 1995). The equations can then be written ignoring the spatial dependence of the fields (but still including an equation for the evolution of the radius). The evolution of a spherical overdense patch of scale radius $R(t)$ is given by the Raychaudhuri equation:

$3 \ddot{R}=-4 \pi G R\left(\rho_{\mathrm{cdm}}+\rho_{\phi_{\mathrm{c}}}\left(1+3 w_{\phi_{\mathrm{c}}}\right)\right)$.

Note that it would be a wrong to use the Friedmann equation for a closed universe with a constant curvature $k$, since the former can vary in time (Weinberg \& Kamionkowski 2003; Wang \& Steinhardt 1998). In the cluster, the evolution of $\rho_{\phi_{\mathrm{c}}}$ and $\rho_{\mathrm{cdm}}$ is given by

$\dot{\rho}_{\phi_{\mathrm{c}}}=-3 \frac{\dot{R}}{R}\left(1+w_{\phi_{\mathrm{c}}}\right) \rho_{\phi_{\mathrm{c}}}+\Gamma$

and $\rho_{\text {cdm }} \propto R^{-3}$ due to mass conservation. The quantity $\Gamma$ describes the energy loss of dark energy inside the dark matter overdensity, as this component does not necessary follow the collapse of dark matter and energy can formally flow out of the system. As such, $\Gamma$ encodes in how far backreaction effects from the dark matter non-linearities act on the dark energy component. Formally, $\Gamma$ can also describe the coupling between matter and dark energy, in which case we would have to add a term containing $\Gamma$ in the energy conservation equation for dark matter. In this case it would be no longer true that $\rho_{\text {cdm }} \propto R^{-3}$.

Once again, in the case of a scalar field, Eq. (11) can be written as

$\ddot{\phi}_{\mathrm{c}}+3 \frac{\dot{R}}{R} \dot{\phi}_{\mathrm{c}}+V_{\mathrm{c}}{ }^{\prime}\left(\phi_{\mathrm{c}}\right)=\frac{\Gamma}{\dot{\phi}}$

where $\phi_{\mathrm{c}}$ is the field inside the overdensity in order to distinguish it from the background value and $V_{\mathrm{c}}=V\left(\phi_{\mathrm{c}}\right)$ is its potential.

We will evolve the spherical overdensity from high redshift until its virialisation occurs. According to the virial theorem, equilibrium will be reached when $T=\frac{1}{2} R \frac{\partial U}{\partial R} ; T=\frac{1}{2} M \bar{v}_{\text {vir }}^{2}$ is the total kinetic energy at virialisation and $\bar{v}_{\text {vir }}^{2}$ is the meansquare velocity of the components of the cluster, and $U$ is the average total potential energy in the sphere. It is useful to write the condition for virialisation to occur in terms of the potential energies associated the different components of the overdensity. The potential energy for a given component " $x$ " can be calculated from its general form in a spherical region (Landau \& Lifshitz 1975):

$$
\begin{aligned}
& U_{x}=2 \pi \int_{0}^{R} \rho_{\mathrm{tot}} \Phi_{x} r^{2} \mathrm{~d} r \\
& \Phi_{x}(r)=-2 \pi G \rho_{x}\left(1+3 w_{x}\right)\left(R^{2}-\frac{r^{2}}{3}\right),
\end{aligned}
$$


where $\rho_{\text {tot }}$ is the total energy density inside the sphere, $\Phi_{x}$ is the gravitational potential due to component $x$ with energy density $\rho_{x}$ and equation of state $w_{x}$. In our calculation, we have taken also the pressure contribution from dark energy into account, since the Poisson-equation reads (including the correction from General Relativity)

$\Delta \Phi=4 \pi G(\rho+3 P)$.

Using the virial theorem and energy conservation at the turnaround and cluster virialisation times, we obtain an equilibrium condition only in terms of the potential energies:

$\frac{1}{2} R \frac{\partial}{\partial R}\left(U_{G}+U_{\phi_{\mathrm{c}}}\right)+U_{G}+\left.U_{\phi_{\mathrm{c}}}\right|_{z_{v}}=U_{G}+\left.U_{\phi_{\mathrm{c}}}\right|_{z_{\mathrm{ta}}}$

where $U_{G}$ is the potential energy for Cold Dark Matter, $U_{\phi_{\mathrm{c}}}$ is the potential energy for dark energy, $z_{\mathrm{v}}$ is the redshift of virialisation and $z_{\mathrm{ta}}$ is the redshift at the turnaround of the overdensity at its maximum radius, when $R=R_{\max }$ and $\dot{R}=0$. In the case where $w_{\phi}=-1$ the expressions reduce to the usual virialisation condition for $\Lambda \mathrm{CDM}$ models. One should point out here the inconsistency widely used in the literature, when one makes use of Eq. (16) together with the assumption that energy is not conserved inside the overdensity (dark energy is assumed to be homogeneous, so it has to flow out from the overdensity). In the case where $\Gamma=0$ the whole process of virialisation is self-consistent.

In addition, we also discuss the linear growth factor $D(z)=$ $\left(\delta_{\mathrm{c}}(z) / a(z)\right) /\left(\delta_{\mathrm{c}}(0) / a(0)\right)$ for the four different potentials. The linear density contrast $\delta_{\mathrm{c}}$ obeys the equation

$\ddot{\delta}_{\mathrm{c}}+2 H \dot{\delta}_{\mathrm{c}}-4 \pi G \rho_{\mathrm{m}} \delta_{\mathrm{c}}=0$.

As we will see, the growth factor $D(z)$ depends on the potential used.

The behaviour of dark energy during the evolution of a cluster can now be obtained by numerically evolving the background Eqs. (3)-(5) and the cluster Eqs. (10)-(12) until the virialisation condition (16) holds. Additionally to the four potentials mentioned above, we also have considered three different types of large scale structure models: the Standard Cold Dark Matter model SCDM, the $\Lambda$ CDM model, and a model with dark energy with constant equation of state $w_{\phi}=$ -0.8 (QCDM). The SCDM and the $\Lambda$ CDM model was used to test our code and we will not give the results for these models, as they are well known.

In next section we will make two assumptions about the function $\Gamma$. In one case we assume that the field is homogeneous all over the Universe, so that the field inside the overdensity evolves just like the background. In the second case we assume that the field collapses together with the dark matter. We would like to point out that our assumptions are different from the ones made in the first version of Lokas \& Hoffman (2001), where it was assumed that dark energy is homogeneous but a Friedmann-like equation was used to study the evolution of $R(t)$.

\section{Non-linear collapse with dark energy}

We now study numerically the equations given in the last section. For this, we must make assumptions about the function $\Gamma$, which encodes the backreaction of dark matter perturbations on dark energy. We will make two assumptions for $\Gamma$ : in the first case, the quintessence field is assumed to be smooth throughout space. This can be obtained by putting

$\Gamma=-3\left(\frac{\dot{a}}{a}-\frac{\dot{R}}{R}\right) \dot{\phi}_{\mathrm{c}}^{2}$

and $\phi_{\mathrm{c}}\left(t_{\mathrm{i}}\right)=\phi\left(t_{\mathrm{i}}\right)$ and $\dot{\phi}_{\mathrm{c}}\left(t_{\mathrm{i}}\right)=\dot{\phi}\left(t_{\mathrm{i}}\right)$. Then we obtain $\phi_{\mathrm{c}}(t)=\phi(t)$ for all times. This is the standard assumption made in the literature. As already mentioned in the introduction, the main reason for this assumption is that the effective Jeans length for linear perturbations turns out to be of the order of the horizon size. Thus, so the argument goes, on scales much smaller than $\lambda_{\mathrm{J}}$ the fluctuations are unimportant.

The form of $\Gamma$ chosen above is clearly not the full truth. At least at very late times during the collapse of the dark matter, especially when the density contrast in dark matter is very large $\left(\delta_{\mathrm{m}} \gg 1\right)$, the field should no longer feel the background metric, i.e. expand with the background, but decouple from it. In this regime, the evolution of dark energy could be different and influence the details of the collapse. The detail of this can only be obtained from a fully relativistic calculation, which is beyond the scope of this paper. However, to get a feel of what may happen we make now the other extreme assumption that the field follows the dark matter collapse from the very beginning. That is, we assume that $\Gamma=0$. This is clearly not a realistic assumption either, but the results we obtain are surprising and interesting in itself.

\subsection{Results for the four quintessence potentials}

We begin our discussion with an analysis of the four different quintessential potentials mentioned in Sect. 2. The results of our numerical calculations are presented in Figs. 1-4.

In these plots we show for all models the behaviour of the equation of state inside the cluster and in the background (for the case $\Gamma=0$ ), the density contrast in matter and field (again for $\Gamma=0$ ), the ratio of the radius at turnaround $R_{\mathrm{ta}}$ and radius at virialisation $R_{V}$ for both the case $\Gamma=0$ and an homogeneous scalar field, the ratio of the energy densities in field and matter, the non-linear density contrast for both cases and the growth factor for the models compared to the $\Lambda \mathrm{CDM}$ model.

As it is clear from the upper right plots, even in the $\Gamma=0$ case (ie. the quintessence field collapses with together with matter), the field will be subdominant in the virialised object. This can also be seen from the Figures on the right hand side in the middle panels in Figs. 1-4: in all cases the ratio $\rho_{\phi} / \rho_{\text {cdm }}$ is much smaller than one. Well before turnaround, i.e. while the density contrast in dark matter is smaller than one, our results agree with what one would obtain from linear perturbation theory. For $\Gamma=0$, the perturbations in dark energy remain very small up to this point. Even after turnaround, our results imply that fluctuations in the dark energy component remain small, while dark matter enters the highly non-linear regime.

Concerning the clustering properties of the quintessence field, it can significantly alter the predictions for the non-linear density contrast $\Delta_{\mathrm{c}}=\rho_{\text {cdm,cluster }}\left(t_{\mathrm{V}}\right) / \rho_{\text {cdm,backgr }}\left(t_{\mathrm{V}}\right)$ at virialisation: assuming that the field is inhomogeneous $\Gamma=0$, 

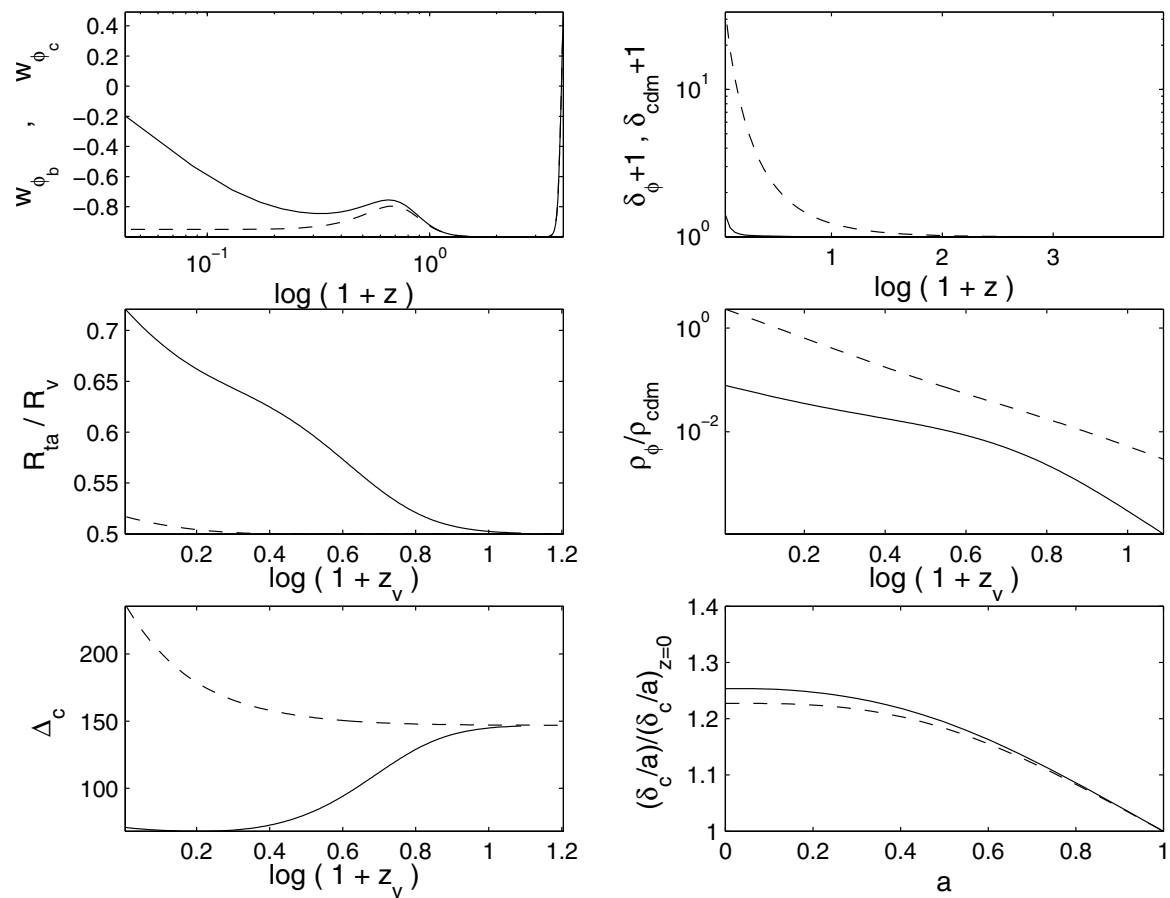

Fig. 1. Quintessence model $V=M(\exp (\beta \phi)+\exp (\gamma \phi))$ (Barreiro et al. 2000). Top left panel: evolution of $w_{\phi}$ in the background (dashed line) and inside an overdensity (solid line) for $\Gamma=0$, as a function of $\log (1+z)$ (overdensity virialises at $z=0$ ). Top right panel: evolution of $\rho_{\phi} / \rho_{\phi_{\mathrm{c}}}$ (solid line) and $\rho_{m} / \rho_{\text {cdm }}$ (dashed line) in the case $\Gamma=0$ as a function of $\log (1+z)$ (overdensity virialises at $z=0$ ). Middle left panel: the ratio $R_{\mathrm{ta}} / R_{\mathrm{V}}$ in the case of an inhomogeneous $(\Gamma=0)$ (solid line) and homogeneous scalar field (dashed line). The ratio $\rho_{\phi} / \rho_{\text {matter }}$ inside the overdensity (solid line) $(\Gamma=0)$ and in the background (dashed line). Bottom left: $\Delta_{\mathrm{c}}$ as a function of $z_{\mathrm{V}}$, considering the effect of an inhomogeneous quintessence field $(\Gamma=0)$ (solid line). The results for a homogeneous quintessence field are shown as well (dashed line). Bottom right: predictions for the linear growth factor for this potential (solid line). The dashed line represents the $\Lambda \mathrm{CDM}$ case.
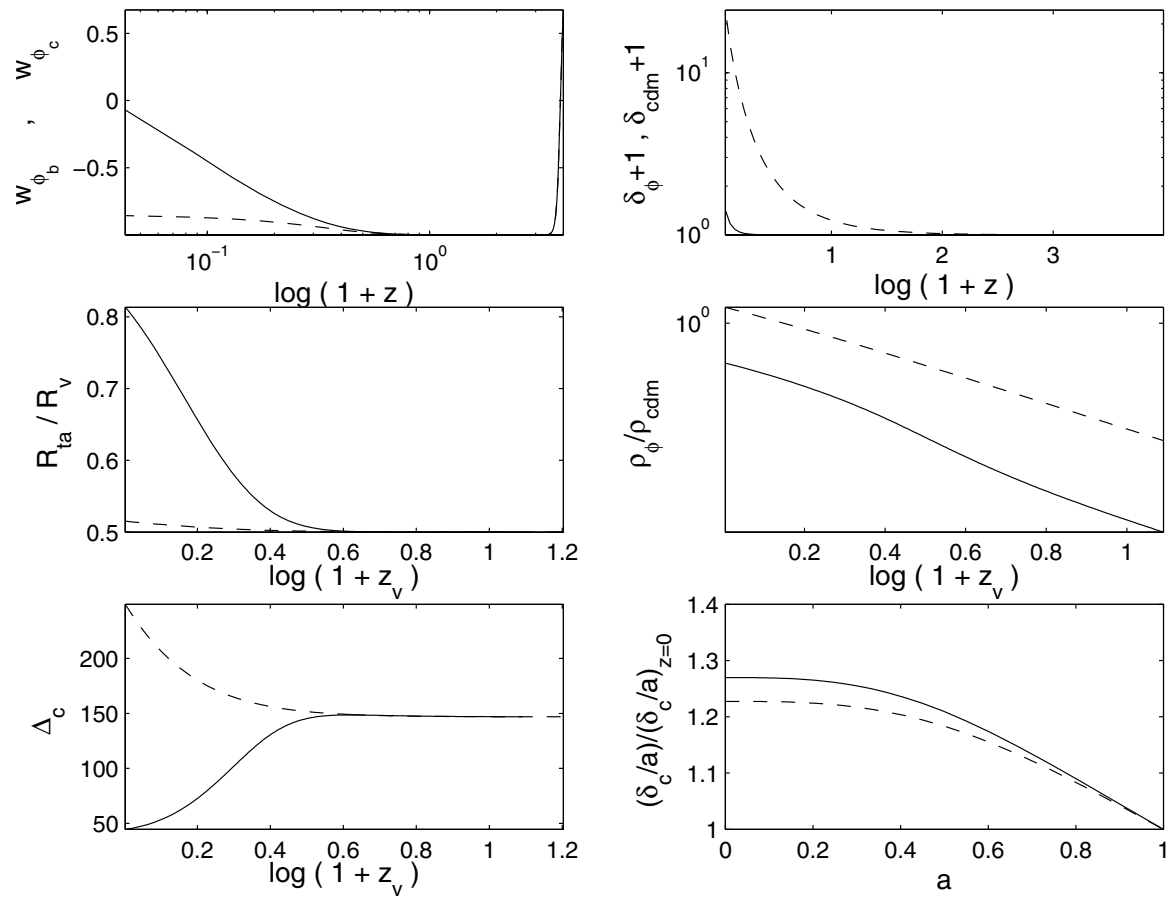

Fig. 2. Quintessence model $V=M(\exp (\gamma / \phi)-1)$ (Steinhardt et al. 1999). Top left panel: evolution of $w_{\phi}$ in the background (dashed line) and inside the overdensity (solid line) for $\Gamma=0$ as a function of $\log (1+z)$ (overdensity virialises at $z=0$ ). Top right panel: evolution of $\rho_{\phi} / \rho_{\phi_{\mathrm{c}}}$ (solid line) and $\rho_{m} / \rho_{\text {cdm }}$ (dashed line) in the case $\Gamma=0$ as a function of $\log (1+z)$ (overdensity virialises at $z=0$ ). Middle left panel: the ratio $R_{\mathrm{ta}} / R_{\mathrm{V}}$ in the case of an inhomogeneous $(\Gamma=0)$ (solid line) and homogeneous scalar field (dashed line). The ratio $\rho_{\phi} / \rho_{\text {matter }}$ inside the overdensity (solid line) $(\Gamma=0)$ and in the background (dashed line). Bottom left: $\Delta_{\mathrm{c}}$ as a function of $z_{\mathrm{V}}$, considering the effect of an inhomogeneous quintessence field ( $\Gamma=0)$ (solid line). The results for a homogeneous quintessence field are shown as well (dashed line). Bottom right: predictions for the linear growth factor for this potential (solid line). The dashed line represents the $\Lambda C D M$ case. 

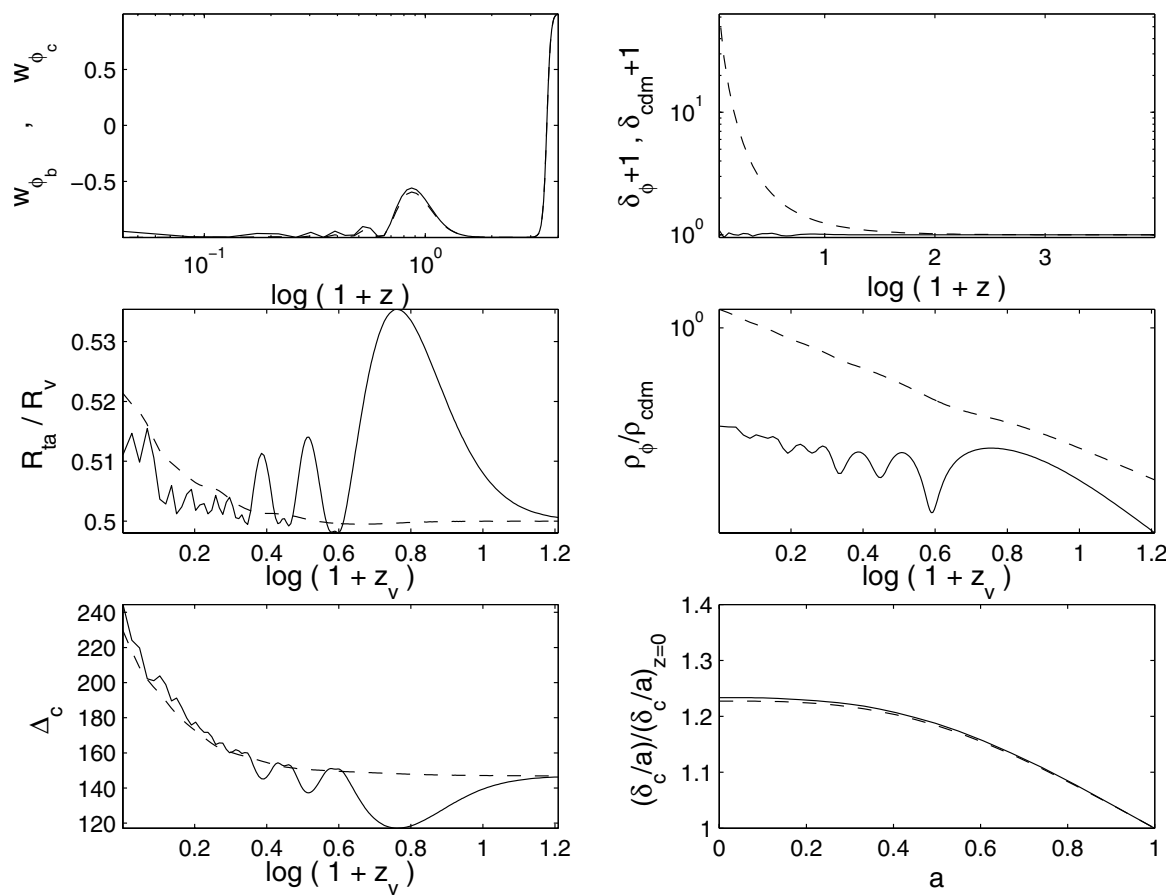

Fig. 3. Quintessence model $V=M\left(A+(\phi-B)^{2}\right) \exp (-\gamma \phi)$ (Albrecht \& Skordis 2000). Top left panel: evolution of $w_{\phi}$ in the background (dashed line) and inside the overdensity (solid line) for $\Gamma=0$ as a function of $\log (1+z)$ (overdensity virialises at $z=0$ ). Top right panel: evolution of $\rho_{\phi} / \rho_{\phi_{\mathrm{c}}}$ (solid line) and $\rho_{m} / \rho_{\text {cdm }}$ (dashed line) in the case $\Gamma=0$ as a function of $\log (1+z)$ (overdensity virialises at $z=0$ ). Middle left panel: the ratio $R_{\mathrm{ta}} / R_{\mathrm{V}}$ in the case of an inhomogeneous $(\Gamma=0)$ (solid line) and homogeneous scalar field (dashed line). The ratio $\rho_{\phi} / \rho_{\text {matter }}$ inside the overdensity (solid line) $(\Gamma=0)$ and in the background (dashed line). Bottom left: $\Delta_{\mathrm{c}}$ as a function of $z_{\mathrm{v}}$, considering the effect of an inhomogeneous quintessence field $(\Gamma=0)$ (solid line). The results for a homogeneous quintessence field are shown as well (dashed line). Bottom right: predictions for the linear growth factor for this potential (solid line). The dashed line represents the $\Lambda C D M$ case.
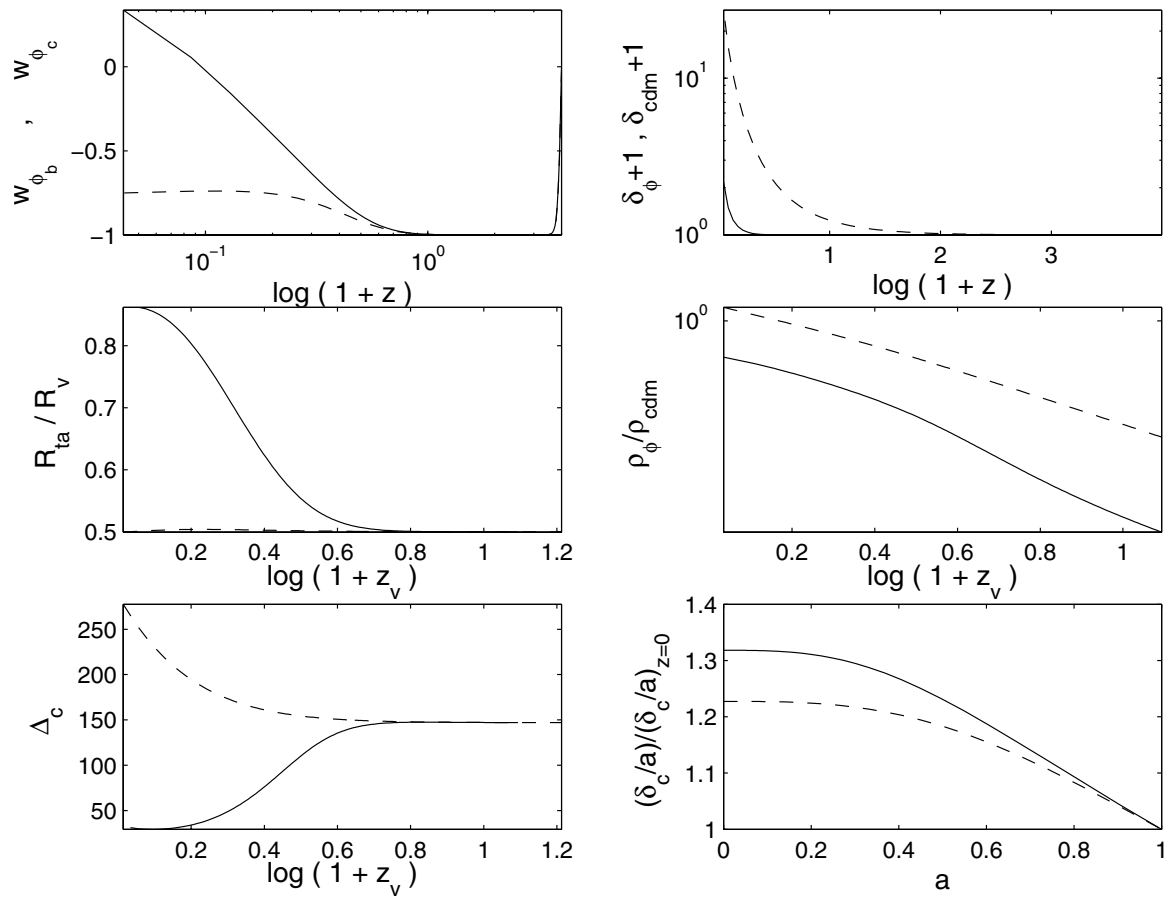

Fig. 4. Quintessence model $V=M \exp \left(\phi^{2}\right) / \phi^{\gamma}$ (Brax \& Martin 1999). Top left panel: evolution of $w_{\phi}$ in the background (dashed line) and inside the overdensity (solid line) for $\Gamma=0$ as a function of $\log (1+z)$ (overdensity virialises at $z=0$ ). Top right panel: evolution of $\rho_{\phi} / \rho_{\phi_{\mathrm{c}}}$ (solid line) and $\rho_{m} / \rho_{\text {cdm }}$ (dashed line) in the case $\Gamma=0$ as a function of $\log (1+z)$ (overdensity virialises at $\left.z=0\right)$. Middle left panel: the ratio $R_{\mathrm{ta}} / R_{\mathrm{V}}$ in the case of an inhomogeneous ( $\Gamma=0)$ (solid line) and homogeneous scalar field (dashed line). The ratio $\rho_{\phi} / \rho_{\text {matter }}$ inside the overdensity (solid line) $(\Gamma=0)$ and in the background (dashed line). Bottom left: $\Delta_{\mathrm{c}}$ as a function of $z_{\mathrm{V}}$, considering the effect of an inhomogeneous quintessence field $(\Gamma=0)$ (solid line). The results for a homogeneous quintessence field are shown as well (dashed line). Bottom right: predictions for the linear growth factor for this potential (solid line). The dashed line represents the $\Lambda C D M$ case. 

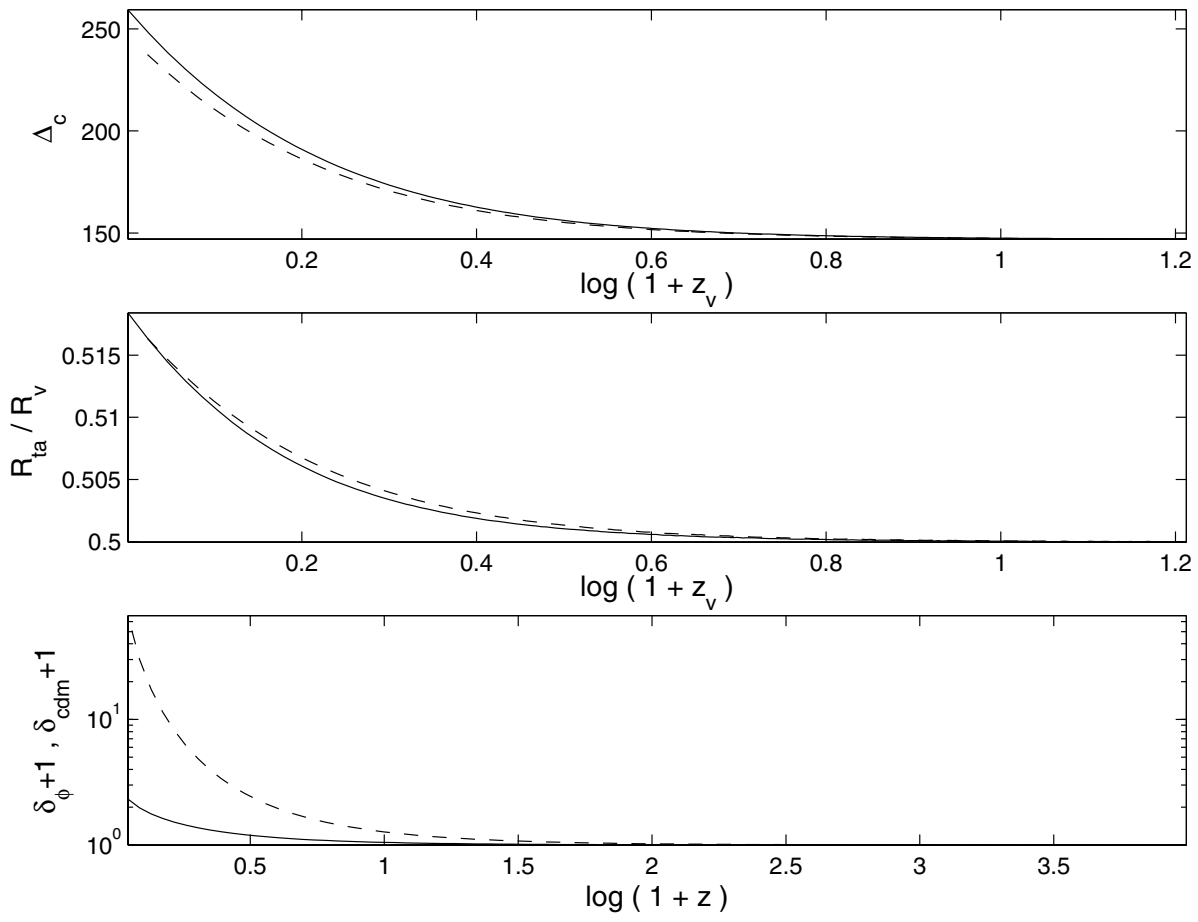

Fig. 5. Model with constant equation of state $w=-0.8$. The upper panel shows the predictions for $\Delta_{\mathrm{c}}$ for the inhomogeneous case (solid line) and homogeneous case (dashed line). The middle panel shows the predictions for $R_{\mathrm{V}} / R_{\mathrm{ta}}$ for the inhomogeneous case (solid line) and homogeneous case (dashed line). The lower panel shows the evolution of the density contrast in matter (dashed line) and dark energy (solid line) for the case of an inhomogeneous dark energy component (cluster virialises at $z=0$ ).

for small virialisation redshifts the predictions can differ by a factor of four or more. In the same way the predictions for the ratio $R_{\mathrm{ta}} / R_{\mathrm{V}}$ strongly depends on how the scalar field behaves during the highly non-linear regime. The reason for this is, that the just before the overdensity formally becomes a singularity, the quintessence field becomes more and more important and changes the evolution of $R(t)$ during the last stages of the collapse. We remind the reader that, in the Einstein-de Sitter model $\Delta_{\mathrm{c}} \approx 147^{3}$ At high virialisation redshifts, all models predict $\Delta_{\mathrm{c}} \approx 147$, only at low virialisation redshifts, significant deviations can be expected due to the fact that the dark energy becomes more and more important. Note that in the Albrecht-Skordis model, the field behaves like a cosmological constant in the background (see Fig. 3). Hence, one would expect that the differences to the $\Lambda$ CDM model are small. This is indeed the case, because $\dot{\phi}_{\mathrm{c}} \approx 0$ and, hence, $\Gamma \approx 0$, as it can be seen from Eq. (18). Thus, in this model, the fluctuations in the quintessence field remain small and the field is almost homogeneous.

In Fig. 5 we have plotted the case for a dark energy model with constant equation of state $w=-0.8$. As it can clearly be seen, the predictions for $\Delta_{\mathrm{c}}$ and $R_{\mathrm{v}} / R_{\mathrm{ta}}$ for the cases of a homogeneous and an inhomogeneous dark energy component are very small in this case. This implies, that for models with constant equation of state and as long as the equation of state

\footnotetext{
${ }^{3}$ If we would define $\Delta_{\mathrm{c} 2}=\rho_{\mathrm{cdm}, \text { cluster }}\left(t_{\mathrm{V}}\right) / \rho_{\mathrm{cdm} \text {,backgr }}\left(t_{\mathrm{c}}\right)$, where $t_{\mathrm{c}}$ is the collapse time, its value in the Einstein-de Sitter model would be approximately 178 . However, for computational convenience, we consider instead the quantity $\Delta_{\mathrm{c}}$ at the time of virialisation. $\Delta_{\mathrm{c}}$ and $\Delta_{\mathrm{c} 2}$ differ only by a factor from each other.
}

doesn't differ too much from $w=-1$, the fitting formulae presented in the literature are valid (Wang \& Steinhardt 1998; Weinberg \& Kamionkowski 2003).

The reason for the differences observed is that in the highly non-linear regime the field will play an important role in determine the collapse time if $\Gamma=0$. Although at the time of virialisation the field is only subdominant, it will play an important role just before collapse. Thus, backreaction effects could significantly alter the predictions of the spherical collapse model.

\subsection{Dependence on the initial conditions}

We turn now our attention to the question, if the results of the spherical model depend on the initial conditions of the field $\phi$ (a discussion of initial conditions in the linear regime can be found in Abramo \& Finelli (2001), Doran et al. (2003), Bartolo et al. (2003)). We have run our code for different initial conditions and will discuss the case for an inhomogeneous $(\Gamma=0)$ and homogeneous dark energy component independently. The case for a homogeneous dark energy component is shown in Fig. 6. In the left upper panel we show the evolution of the equation of state in the background for different initial values for $\phi$ at a redshift of $10^{4}$. As it can be seen, the ratio $\rho_{\phi} / \rho_{\text {cdm,clus }}$ is almost independent of the initial conditions chosen. The same is true for the predictions of $\Delta_{\mathrm{c}}$ and $R_{\mathrm{ta}} / R_{\mathrm{v}}$.

The case for $\Gamma=0$ is shown in Fig. 7. We find that if quintessence becomes inhomogeneous together with dark matter in the strong non-linear regime, the predictions of the spherical collapse model depend generically on the initial conditions of the field. 

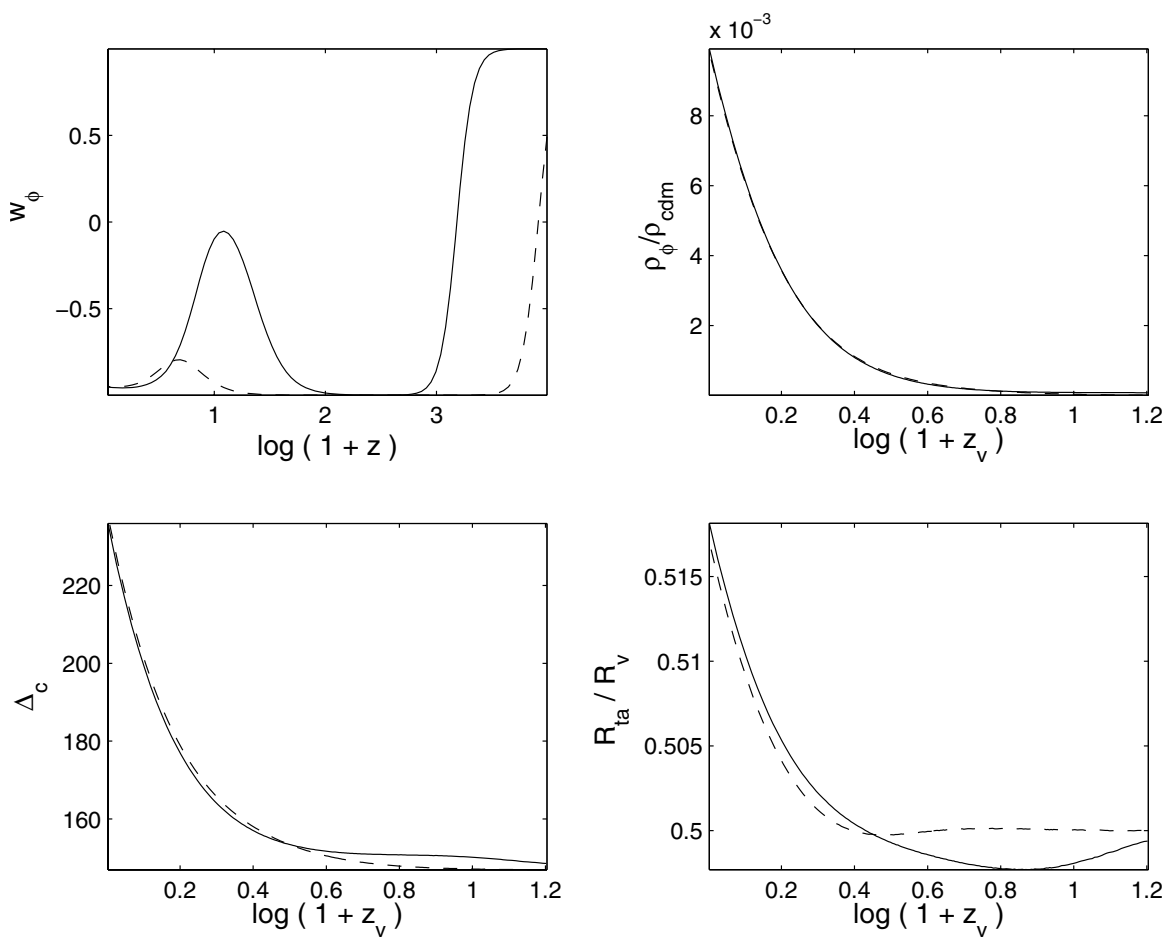

Fig. 6. Dependence on the initial conditions for the double exponential potential (Barreiro et al. 2000) for the case of homogeneous dark energy. The solid and dashed lines show the results for different initial conditions. Top left panel: evolution of $w_{\phi}$ in the background as a function of $\log (1+z)$. Top right panel: the radio $\rho_{\phi} / \rho_{\text {matter }}$ inside the overdensity as a function of $\log \left(1+z_{\mathrm{v}}\right)$. Bottom left: evolution of $\Delta_{\mathrm{c}}$ as a function of $\log \left(1+z_{\mathrm{v}}\right)$. Bottom right: evolution of $R_{\mathrm{ta}} / R_{\mathrm{v}}$ as a function of $\log \left(1+z_{\mathrm{v}}\right)$. Whereas the evolution of the equation of state is different, the predictions for $\rho_{\phi} / \rho_{\mathrm{cdm}}, \Delta_{\mathrm{c}}$ and $R_{\mathrm{ta}} / R_{\mathrm{v}}$ are only weakly dependent on the initial conditions.
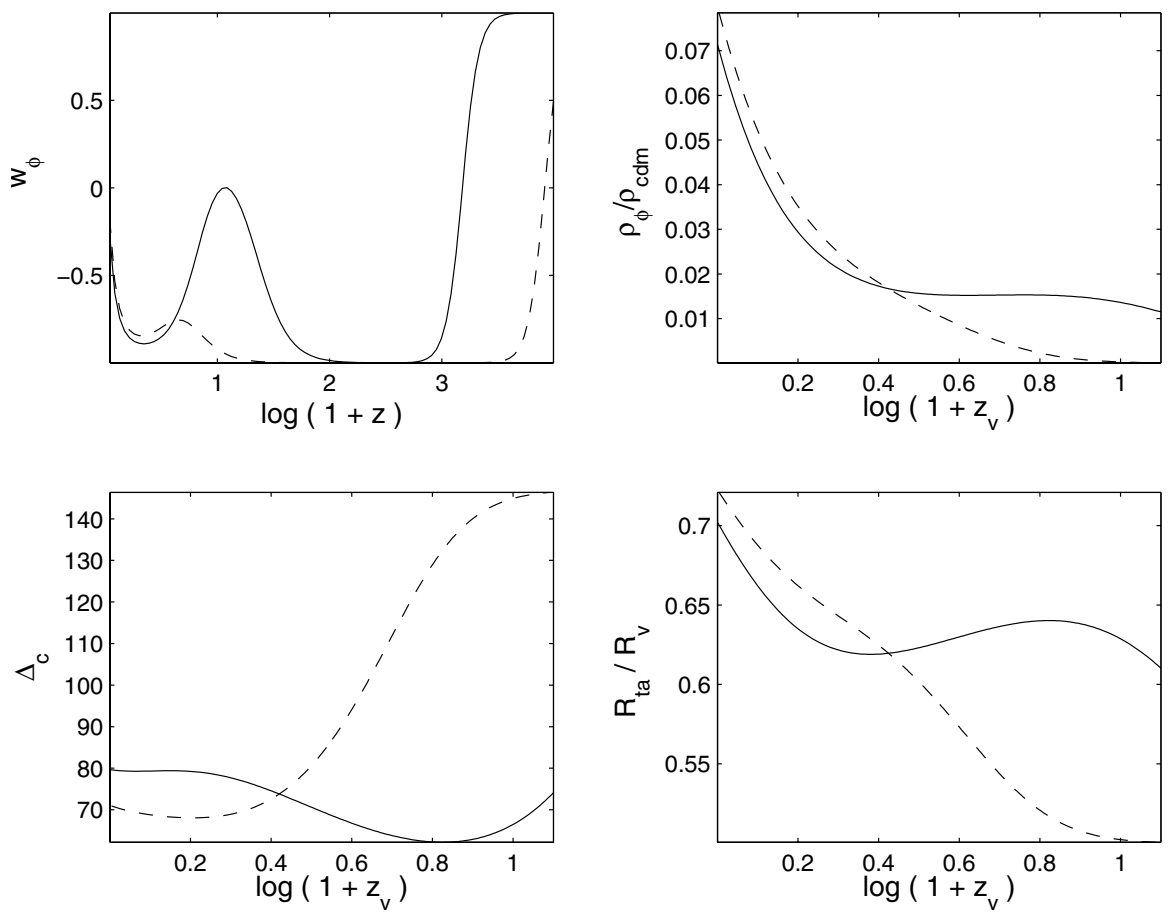

Fig. 7. Dependence on the initial conditions for the double exponential potential (Barreiro et al. 2000) in the case for an inhomogeneous dark energy component $(\Gamma=0)$. The solid and dashed lines show the results for different initial conditions. Top left panel: evolution of $w_{\phi}$ in the overdensity as a function of $\log (1+z)$. Top right panel: the radio $\rho_{\phi} / \rho_{\text {matter }}$ inside the overdensity as a function of $\log \left(1+z_{\mathrm{v}}\right)$. Bottom left: evolution of $\Delta_{\mathrm{c}}$ as a function of $\log \left(1+z_{\mathrm{v}}\right)$. Bottom right: evolution of $R_{\mathrm{ta}} / R_{\mathrm{v}}$ as a function of $\log \left(1+z_{\mathrm{v}}\right)$. It can clearly be seen that the predictions in this case depend on the initial conditions. 

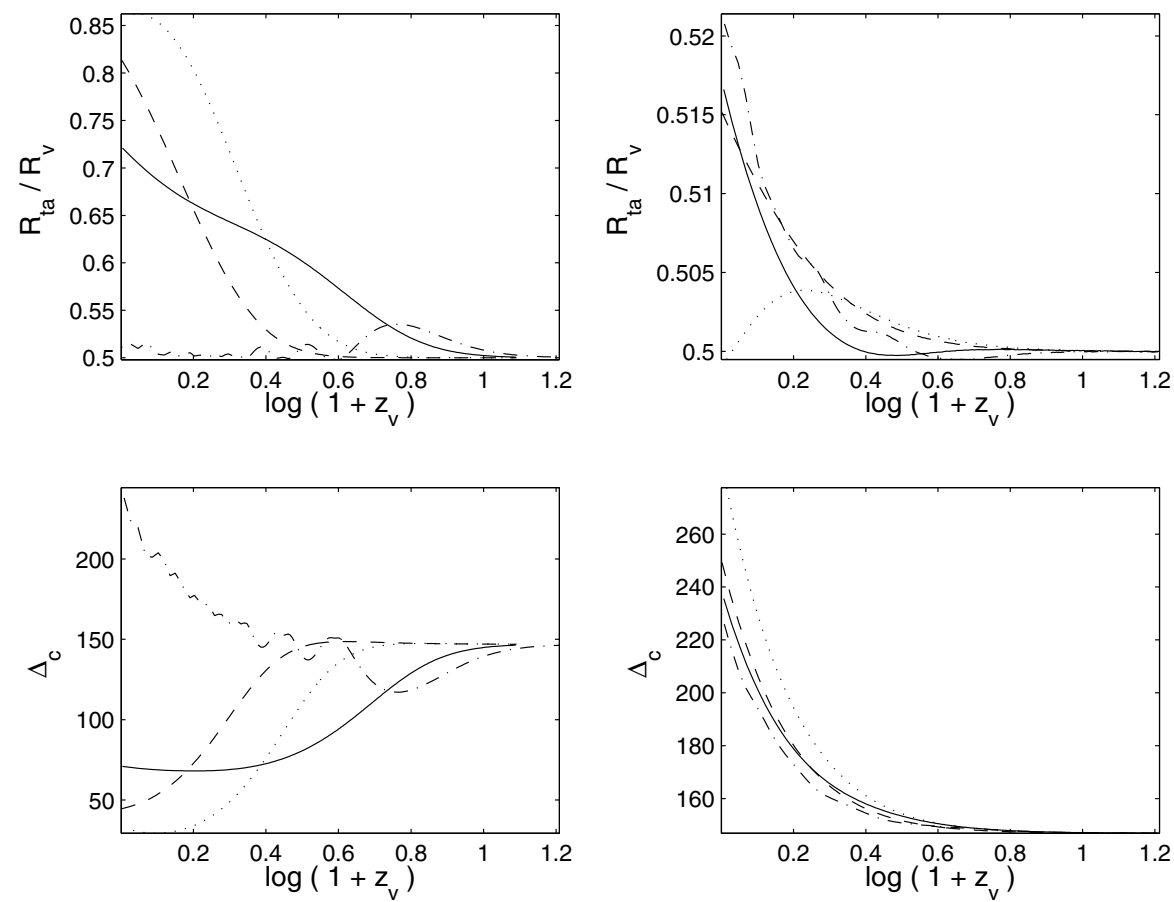

Fig. 8. The predictions for $R_{\mathrm{ta}} / R_{\mathrm{v}}$ and $\Delta_{\mathrm{c}}$ for the different quintessential potentials. The left panels show the case for an inhomogeneous dark energy component $(\Gamma=0)$, whereas the right panel shows the results for the case of an homogeneous dark energy component. It can be seen that the predictions depend on the potential used, although the dependence is weaker in the case of an homogeneous dark energy component. Solid line: Barreiro et al. (2000), dashed-line: Steinhardt et al. (1999), dashed-dotted line: Albrecht \& Skordis (2000), dotted-line: Brax \& Martin (1999).

Different initial conditions lead to different time evolution of the equation of state and the energy density of quintessence, both in the background and inside the overdensity (depending on the value of $\Gamma$ ). These two dark energy properties will affect the dynamics of the overdensity through Eqs. (10) and (14), hence affecting the predictions for the non-linear density contrast and the virialisation radius of the overdensity.

\subsection{Comparison among the different models}

What are the differences in the predictions for the four quintessence models? In Fig. 8 we plot the predictions for $R_{\mathrm{ta}} / R_{\mathrm{v}}$ and $\Delta_{\mathrm{c}}$ for the different potentials with the different assumptions for $\Gamma$. As it can be seen from that figure, the differences can be quite large if $\Gamma=0$. If the field is homogeneous throughout space, the differences are small. Again, the reason is that the dynamics of the overdensity (see Eqs. (10) and (14)) strongly depends on the dark energy properties (Potential, $\Gamma$, etc.).

In Fig. 9 we plot the predictions of the linear growth factor $\left(\delta_{\mathrm{c}} / a\right)$, normalised today, for the different potentials. As it can be seen, even in the linear regime there are differences between the four potentials. Such a behaviour was already noticed in Maccio et al. (2003a).

\subsection{Delayed collapse of dark energy}

The results presented imply that if dark energy collapses together with dark matter, it has an important impact on the

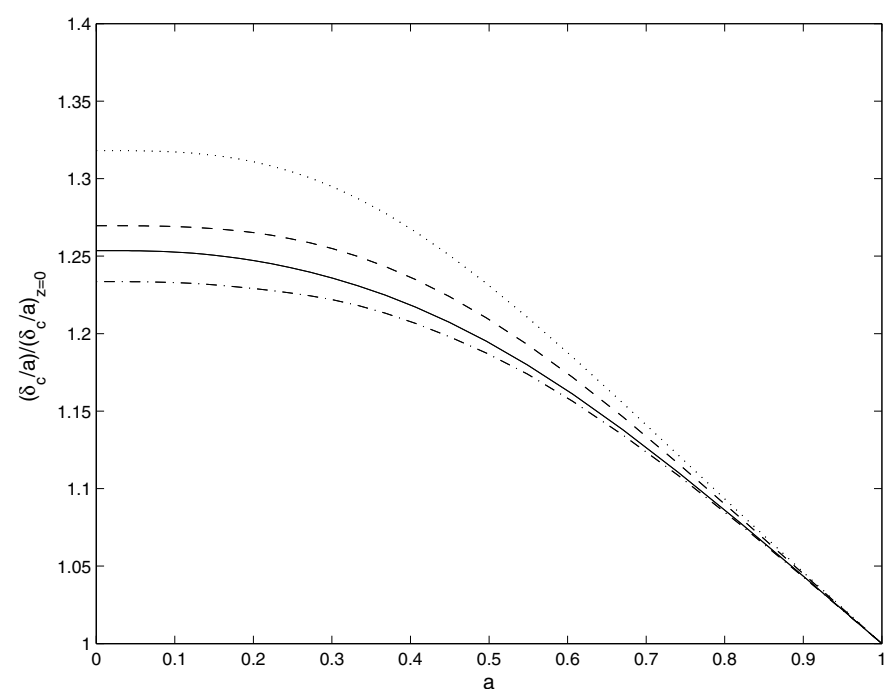

Fig. 9. The linear growth factor for the different quintessential potentials. It can be seen that the growth factor depends on the potential used. Solid line: Barreiro et al. (2000), dashed-line: Steinhardt et al. (1999), dashed-dotted line: Albrecht \& Skordis (2000), dotted-line: Brax \& Martin (1999).

predictions of the spherical collapse model. Clearly, our assumption on the parameter $\Gamma$ so far is unrealistic. To gain more insight in how far our choices of $\Gamma$ changes the predictions of the spherical collapse model, we make now the assumption that the field inside the cluster is the same as in the background until the turnaround, from which on it collapses together with the dark matter. We will call this situation as the delayed collapse 

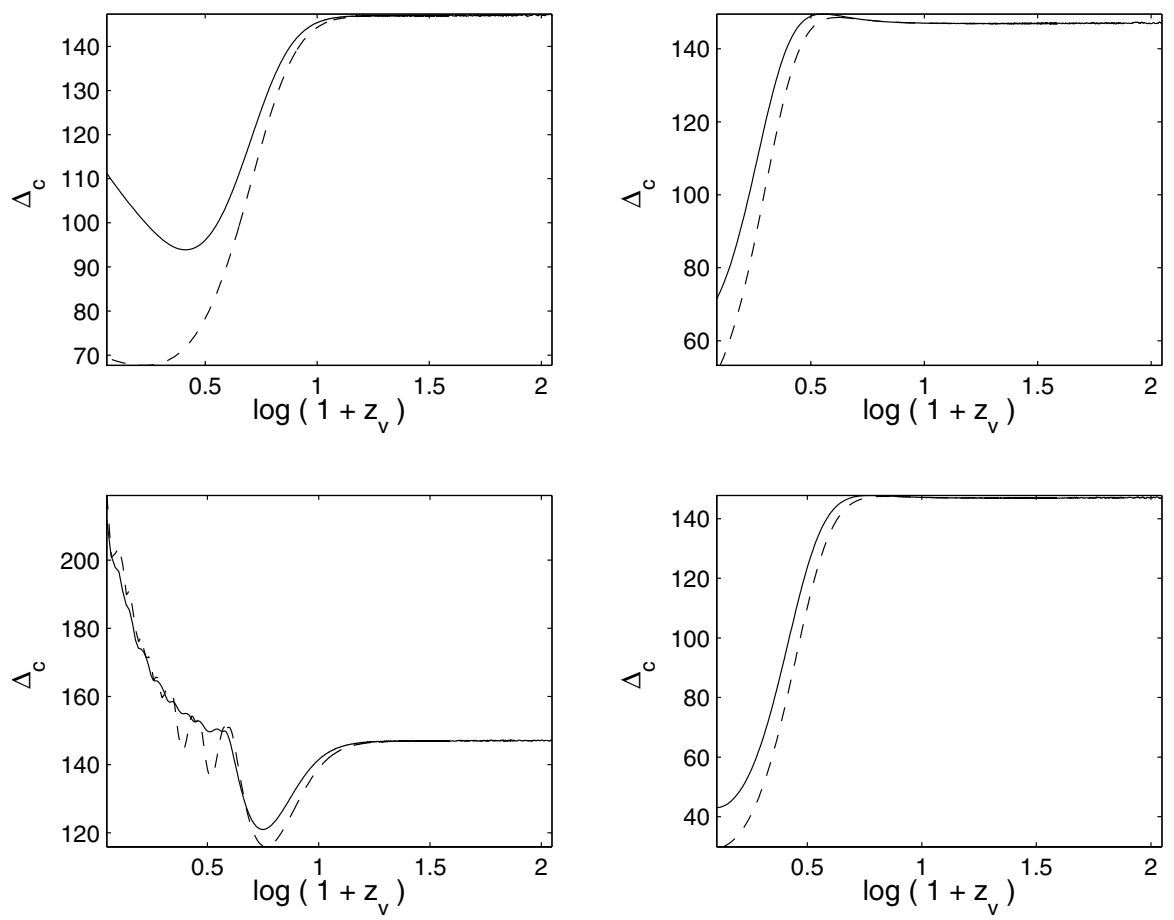

Fig. 10. Predictions for $\Delta_{c}$ in the case of delayed collapse of dark energy. In all four panels, the solid line corresponds to the delayed collapse situation, the dashed line to the case of $\Gamma=0$ both before and after turnaround. The upper left panel shows the predictions for the double exponential (Barreiro et al. 2000), the left upper panel for the model of Steinhardt et al. (1999), the lower left panel the model of Albrecht \& Skordis (2000) and the lower right panel the model of Brax \& Martin (1999).

of dark energy. In the light of results obtained in the linear theory (see e.g., Ferreira \& Joyce 1997), this is a more realistic situation than studied up to now. The results for the precictions of $\Delta_{c}$ are shown in Fig. 10.

In Fig. 10 we show the results for $\Delta_{c}$ for all four potentials. The solid lines are the cases for delayed collapse of dark energy, whereas the dashed lines correspond to the case of full collapse. As it can be clearly seen, the predictions for $\Delta_{c}$ change. Note, however, that the predictions are still far off from the the results obtained in the case of a homogeneous scalar field. Of course, the field is in the linear regime, but it has still an important impact on the predictions of the spherical collapse model.

\section{Conclusions}

In this paper we have studied the spherical collapse model in cosmologies with dark energy, provided by a scalar field (quintessence). Our aim was to study the model for different quintessence potentials and under different assumptions about the behaviour of the field during the highly non-linear regime. Our results show, that the predictions of the spherical collapse model depend on the form of the potential, on the initial conditions of the field and on the behaviour of the field in highly non-linear regions. If backreaction effects onto the field turn out to be important, these could significantly change the predictions of the spherical collapse model.

We would like to point out that although the dark matter enters the highly non-linear regime first, perturbations in dark energy deviate only slightly from linear theory around virialisation. At the time of virialisation, the energy density of dark energy is always much smaller than the density of dark matter inside an overdensity. The main effect is that dark matter perturbations change the evolution of dark energy perturbations. The physical properties of clusters, such as the density contrast and virial radius strongly depend on clustering properties of dark energy (i.e. the parameter $\Gamma$ ), the quintessence potential and the initial conditions for the field fluctuations. The latter is only important, however, if the field collapses together with the dark matter. On the other hand, we have found that if the dark energy equation of state is assumed to be constant, the differences between the homogeneous and inhomogeneous cases are small, at least if the equation of state $w$ does not differ too much from $w=-1$. Thus, for constant equation of state the fitting formulae presented in the literature (see Wang \& Steinhardt 1998; Weinberg \& Kamionkowski 2003) do not change drastically even if inhomogeneities in the dark energy component are taken into account.

Our results imply, that a better understanding of the behaviour of the quintessence field in highly non-linear regions is needed before the predictions of the spherical collapse model can be trusted. The field can influence the dynamics of the collapse at late times and thus changing predictions of the turnaround, virialisation and collapse times. In order to get better predictions, the boundary conditions between the outer and inner metric have to be understood better. Its likely that the spherical model just gives qualitative but not quantitative predictions. Nevertheless, an estimate of the function $\Gamma$ is needed, which can only be obtained from a general relativistic treatment. One way would be to develop a swiss cheese model in 
the case of a quintessential universe. This will be discussed in future work.

Our calculations have also implications for models in which the scalar field couples non-minimally to dark matter. In these models, the scalar field perturbations and the dark matter perturbations are coupled even in the linear regime and backreaction in the highly non-linear regime will certainly be important. A similar conclusion might hold for models based on tachyonic fields.

To summarise, only when the energy flux of scalar field energy density $\Gamma$ out of a dark matter overdensity is known, the spherical collapse model is able to make firm predictions which can be used to make predictions for weak and strong lensing, the number density of clusters, etc or even for a help to use $N$-body simulations in dark energy models. In the case of a non-minimally coupled dark energy component, the predictions of the spherical collapse model will certainly be altered.

Acknowledgements. We would like to thank P. G. Ferreira, J. Magueijo, K. Moodley, C. Skordis, J. Silk and D. Tocchini-Valentini, for helpful comments. D.F.M. is supported by Funda cão Ciência e a Tecnologia. C.v.d.B. is supported by PPARC.

\section{References}

Abramo, L. R. W., \& Finelli, F. 2001, Phys. Rev. D, 64, 083513

Albrecht, A., \& Skordis, K. 2000, Phys. Rev. Lett., 84, 2076

Alcubierre, A., Guzman, F., Matos, T., et al. 2002, Class. Quant. Grav., 19,5017

Amendola, L. 2000, Phys. Rev. D, 62, 043511

Amendola, L. 2003, Linear and Non-Linear Perturbations in Dark Energy Models [arXiv:astro-ph/0311175]

Arbey, A., Lesgourgues, J., \& Salati, P. 2001, Phys. Rev. D, 64, 123528

Bagla, J., Jassal, H., \& Padmanabhan, T. 2003, Phys. Rev. D, 67, 063504

Bartelmann, M., Perrotta, F., \& Baccigalupi, C. 2002, A\&A, 396, 21

Bartolo, N., Corasaniti, P., Liddle, A. R., \& Marquarti, M. 2003, Perturbations in cosmologies with scalar field and a perfect fluid [arXiv: astro-ph/0311503]

Battye, R. A., \& Weller, J. 2003, Phys. Rev. D, 68, 083506

Brax, P., \& Martin, J. 1999, Phys. Lett. B, 468, 40

Barreiro, T., Copeland, E., \& Nunes, N. J. 2000, Phys. Rev. D, 61 127301

Bean, R., \& Magueijo, J. 2002, Phys. Rev. D, 66, 063505

Caldwell, R. R., Dave, R., \& Steinhardt, P. 1998, Ap\&SS, 261, 303

Causse, M. 2003, A rolling tachyon field for both dark energy and dark halos of galaxies [arXiv: astro-ph/0312206]

Dolag, K., Bartelmann, M., Perrotta, F., et al. 2003, Numerical Study of Halo Concentrations in Dark-Energy Cosmologies [arXiv: astro-ph/0309771]
Doran, M., Mueller, C., Schaefer, G., \& Wetterich, C. 2003, Phys. Rev. D, 68, 063505

Ferreira, P. G., \& Joyce, M. 1997, Phys. Rev. Lett., 79, 4740

Giovi, F., Baccigalupi, C., \& Perrotta, F. 2003, Weak lensing, structure formation and dark energy [arXiv: astro-ph/0309422]

Guzman, F., \& Urena-Lopez, L. 2003, Phys. Rev. D, 68, 024023

Hwang, J. C., \& Noh, H. 2001, Phys. Rev. D, 64, 103509

Lahav, O., Rees, M. J., Lilje, P. B., Primack, J. R., et al. 1991, MNRAS, 251, 128

Landau, L., \& Lifshitz, E. 1975, The Classical Theory of Fields, 4th. ed. (Oxford: Pergamon)

Linder, E. V., \& Jenkins, A. 2003, MNRAS, 346, 573

Lokas, E., \& Hoffman, Y. 2001, Nonlinear evolution of spherical perturbation in the universe with cosmological constant and quintessence [arXiv: astro-ph/0108283]

Ma, C., Caldwell, R. R., Bode, P., \& Wang, L. 1999, ApJ, 521, L1

Maccio, A. V., Bonometto, S. A., Mainini, R., \& Klypin, A. 2003a, Structure formation in dynamical dark energy models [arXiv: astro-ph/0309439]

Maccio, A. V., Quercellini, C., Mainini, R., Amendola, L., \& Bonometto, S. A. 2003b, $N$-body simulations for coupled dark energy: Halo mass functions and density profiles [arXiv:astro-ph/0309671]

Mainini, R., Maccio, A. V., \& Bonometto, S. A. 2003a, New Astron., 8,173

Mainini, R., Maccio, A. V., Bonometto, S. A., \& Klypin, A. 2003b, ApJ, 599, 24

Matarrese, S, Pietroni, M., \& Schimd, C. 2003, JCAP, 0308, 005

Mota, D. F., \& Barrow, J. D. 2004a, MNRAS, 349, 281

Mota, D. F., \& Barrow, J. D. 2004b, Phys. Lett. B, 581, 141

Padmanabhan, T. 1995, Structure formation in the universe (Cambridge University Press)

Padmanabhan, T., \& Choudhury, T. 2002, Phys. Rev. D, 66, 081301

Padmanabhan, T. 2002, Phys. Rev. D, 66, 021301

Peacock, J. 1999, Cosmological Physics (Cambridge University Press)

Perlmutter, S., Aldering, G., Goldhaber, G., et al. 1999, ApJ, 517, 565

Perrotta, F., Matarrese, S., Pietroni, M., \& Schimd, C. 2004, Phys. Rev. D, 69, 084004

Ratra, B., \& Peebles, P. 1988, ApJ, 325, L17

Ratra, B., \& Peebles, P. 2002, J. Rev. Mod. Phys., 75, 559

Steinhardt, P. J., Wang, L., \& Zlatev, I. 1999, Phys. Rev. D, 59, 123504

Steinhardt, P. 2003, Phil. Trans. Roy. Soc. Lond. A, 361, 2497

Tonry, J., Schmidt, B. P., Barris, B., et al. 2003, ApJ, 594, 1

Wang, L. M., \& Steinhardt, P. J. 1998, ApJ, 508, 483

Weinberg, N., \& Kamionkowski, M. 2003, MNRAS, 341, 251

Weller, J., Battye, R., \& Kneissl, R. 2002, Phys. Rev. Lett., 88, 231301

Wetterich, C. 1988a, Nucl, Phys. B, 302, 645

Wetterich, C. 1988b, Nucl. Phys. B, 302, 668

Wetterich, C. 1995, A\&A, 301, 321

Wetterich, C. 2001, Phys. Lett. B, 522, 5

Wetterich, C. 2002, Phys. Rev. D, 65, 123512 